\title{
CORRELATE BETWEEN BIOFILM FORMATION AND AZOLE ANTIFUNGAL SUSCEPTIBILITY AGAINST PLANKTONIC AND SESSILE CANDIDA ALBICANS CLINICAL ISOLATES
}

Raja Kamran Afzal*, Shahzad Hassan**, Aamir Ikram**, Saira Salim*, Amir Hussain, Maria Khan

Combined Militar Hospital Peshawar/National University of Medical Sciences (NUMS) Pakistan, *Khyber Medical University, Peshawar Pakistan

\section{ABSTRACT}

Objective: To assess the correlation between biofilm formation and azole antifungal susceptibility against plank tonic and sessile clinical isolates of C.albicans.

Study Design: Prospective observational study.

Place and Duration of Study: Combined Military Hospital Peshawar, from Jun 2016 to Sep 2017.

Methodology: All standard microbiological procedures were carried out according to latest Clinical \& laboratory standard institute (CLSI) guidelines. After gram staining and presumptive identification on CHRO Magar Candida, the isolates were biochemically identified by API AUX Candida as C.albicans. Planktonic antifungal susceptibility was carried out by Kirby Bauer disk diffusion method on 300 C.albicans isolates. Broth microdilution method was used to determine Minimum inhibitory concentration (MICs) of plank tonic cells and micro titer assay was used for assessment of biofilm formation by C.albicans. Results: In planktonic antifungal susceptibility, fluconazole was susceptible against 195 (65\%) and voriconazole against 241 $(80 \%)$ C. albicans isolates. C. albicans was found susceptible dose dependent (SDD) to fluconazole in $28(9 \%)$ and to voriconazole in $21(7 \%)$ isolates. Seventy-seven $(26 \%)$ and $38(13 \%)$ C.albicans isolates were found fluconazole and voriconazole resistant, respectively. Sessile antifungal susceptibility was carried out through broth micro dilution method in which 160 (53\%) were susceptible, $42(14 \%)$ were susceptible dose dependent SDD and $98(33 \%)$ were resistance to voriconazole, and $161(54 \%)$ were susceptible, $36(12 \%)$ were SDD and $103(34 \%)$ were found resistant to fluconazole. Biofilm forming isolates of C.albicans were observed to be $285(95 \%)$.The $p$-value is highly significance i.e. $<0.01$ between the biofilm formation and azole antifungal susceptibility against plank tonic and sessile clinical isolates of C.albicans.

Conclusion: Plank tonic C.albicans clinical isolates appeared more susceptible to voriconazole than fluconazole and sessile isolates. Biofilm formation was very high among all the isolates of C.albicans.

Keywords: Azole antifungals, Biofilm formation, Broth microdilution, Candida albicans, Disk diffusion.

This is an Open Access article distributed under the terms of the Creative Commons Attribution License (https://creativecommons.org/licenses/by-nc/4.0/), which permits unrestricted use, distribution, and reproduction in any medium, provided the original work is properly cited.

\section{INTRODUCTION}

The genus Candida is a dimorphic fungus, means that develops as bothfilamentous and yeast. It has $>150$ species but only fewpathogenic to human, and it is responsible for about $50-90 \%$ all candidiasis in human ${ }^{1}$. Candida albicans is the commonest among these. It is a part of human intestinal flora and is foundin, gastrointestinal tract in $40 \%$ of healthy adults. Candidais the leading cause of opportunistic fungal infections in human².

Systemic fungal infection causedby C.albicans has developed ${ }^{3}$, an vital cause of, morbidity and mortality in immocompromised patients of AIDS, organ or bone marrow transplantation and cancer chemotherapy ${ }^{4}, \mathrm{C}$. albicans biofilms may establish on the surface of implanted medical devices, more so in immunocompromised hosts 5 . These include commonly the fungal infections on any type of implanted catheter, removable devices like dentures, voice prosthesis and contact

Correspondence: Dr Saira Salim, House No. B-314/25, Officer Colony, Wah Cantt Pakistan

Received: 28 Aug 2020; revised received: 17 Dec 2020; accepted: 21 Dec 2020 lensrelatedfungal keratitis ${ }^{6}$.

Candidiasis is of three main types,oropharyngeal or an invasive candidiasis like esophageal and genital or vulvo vaginal. Oropharyngeal candidiasis is also called thrush, candidiasis in the vagina is called yeast infection and invasive candidiasis appear when it goes to the bloodstream and tends to spread systemically ${ }^{7}$.

Biofilms are groups of microbes found on surfaces, which can be spotted in medical, natural and industrial settings ${ }^{8}$. Biofilm production by C.albicans is a complicated process and comprises of three important steps in the process of development. Biofilm is characterized by, attachment of the yeast blastopores colonization on the substrate expansion of the yeast cells, followed by the formation of the biofilm colonization on the substrate expansion of the yeast cells, followed bytheformation of the biofilm ${ }^{9,10}$.

Novel technological approaches have been devised to study the formation of biofilms with identification of their markers. Biofilms show reduced susceptibility to most of antimicrobial agents leading to the persistence of infection within it. Due to formation of an extra cellular framework the cells are saved from 
the environment and it becomes more difficult for antifungal to approach the yeast cells ${ }^{11}$.

Against most antifungal agents except the lipid formulations of amphotericin B and echinocandins, Candida biofilms possess a multi-factorial broad-spectrum defense mechanisms ${ }^{12}$. During the early phase of formation of biofilm, the sterol composition of membrane is altered and the efflux pumps of drugs are expressed, thus contributing to the antifungal resistance against azoles ${ }^{13}$.

The objective of this study is that the correlation between the biofilm formations by C.albicans isolated from clinical specimens and azole antifungal susceptibility against its planktonic and sessile forms.

\section{METHODOLOGY}

It was prospective analytical study, all clinical specimens from indoor and outdoor patients submitted to Combined Military Hospital (CMH) PeshawarKPK for fungal culture and susceptibility testing, from September 2016 to May 2017 were included. Non-probability consecutive sampling technique was used for specimen collection. Sample size was 300 according to WHO calculator ${ }^{14}$. Repeat and inadequate specimens and Candida non-albicans isolates were excluded.

\section{Fungal Culture}

For obtaining a fungal growth, whole specimens were cultured aerobically on SaboraudDextrose agar (SDA) (Oxoid $\left.{ }^{\mathrm{TM}}\right)$ for $24-48$ hours at $37^{\circ} \mathrm{C}$. After a growth was available, Gram stain was done for preliminary identification of the yeast. For presumptive identification, the isolates were inoculated on CHRO Magar TM Candida (Oxoid $\left.{ }^{\mathrm{TM}}\right)$, which is a selective and a differential medium having chromogenic substrate, and where Candida appeared as green-colored colonies. API $20 \mathrm{C}$ AUX (bio Mérieux, France) was used to differentiate biochemically C.albicans from other Candida species.

\section{Antifungal Susceptibility Testing}

Antifungal susceptibility testing against planktonic C. albicans was undertaken by Kirby Bauer disc diffusion procedure. A 0.5 McFarland standard suspension was prepared from a $24-48$ hours old culture of $C$. albicans. The isolate was streaked on the plate of Mueller Hinton agar (MHA) (Oxoid $\left.{ }^{\mathrm{TM}}\right)$. Using Disc dispenser (Oxoid $\left.{ }^{\mathrm{TM}}\right)$, fluconazole $(25 \mu \mathrm{g})$ andvoriconazole $(5 \mu \mathrm{g})$ antifungal discs (Oxoid ${ }^{\mathrm{TM}}$ ) were applied on the surface of MHA according to CLSI M44A ${ }^{8}$. These plates were kept at $35^{\circ} \mathrm{C}$ and observed for growth after 24 to 48 hours, followed by measurement of zones of inhibition in millimeters for the antifungal discs applied. The antifungal susceptibility (susceptible $S$, susceptible dose-dependent SDD, and resistant R) was interpreted as per CLSI standards9. C. albicans ATCC 64548 strain was used to ensure quality control. Minimum inhibitory concentration (MIC) of both the azoles against C.albicans isolates was carried out in a 96-well roundbottom microtiter plate in RPMI 1640 -L-glutamines (Thermo Scientific TM) according to CLSI M27 Yeast ${ }^{10}$. The microdilution plates hadcoved at $35^{\circ} \mathrm{C}$ and were seen for visibility of any growth (fig-1). The microdilution wells were scored with, the aid of a reading mirror, the growth in every well was matched with that of the growth control (drug-free) well. After 24-48 hours clearing in turbidity was observed through the lens.

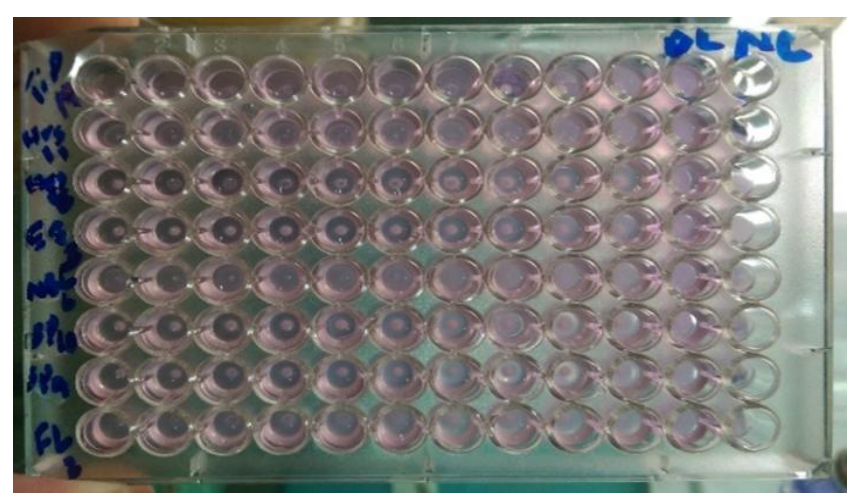

Figure-1: Optical clearness of turbidity in different wells of the microtiter plate.

\section{Biofilm Formation}

For biofilm formation, unmixed culture of $C$. albicans was developed on sabourd agar at $37^{\circ} \mathrm{C}$ for 24 hours. Sterile (96) well flat bottom microtiter plate was consumed. Four wells were used for every sample. The left well was used for negative control andtheright one was used as positive control. RPMI 1640-L-glutamines (Thermo Scientific TM) was included to every selected wells in the form of series. A $50 \mu 1$ inoculum of every sample was included in columns and $50 \mu$ l of human serumwas included in the selected well. This microtiter plate was set in a vibrating incubator for 24 hours at $37^{\circ} \mathrm{C}$ and biofilm production was observed (fig-2).

This plate was washed with approximately $100 \mu 1$ PBS (phosphate buffer saline) after 24 hours of incubation at $37 \mathrm{C}$ and $100 \mu \mathrm{l}$ of a $0.1 \%$ solution of crystal violet $(\mathrm{CV})$ in water were included in every well. This plate included at room temperature for 10-15 min and washed twice. It was turned upside down and left to dry for a few hours. About $100 \mu 1$ of $30 \%$ acetic acid in water was included inevery well of the microtiter plate to solubilize CV and it was incubated at room temperature for $10-15 \mathrm{~min}$. Solubilized CV $100 \mu \mathrm{l}$ was put to a 
new flat bottom microtiter dish. Absorbance was quantified in a plate reader (Bio Tek-Elx800) at $620 \mathrm{~nm}$.

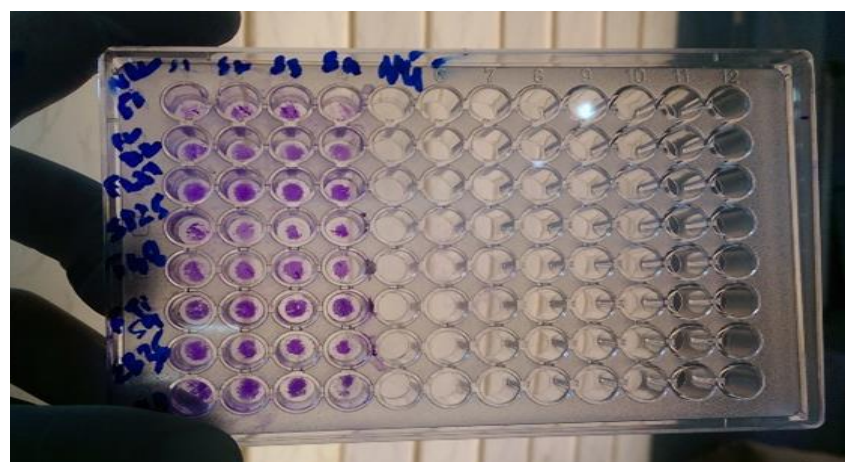

Figure-2: Biofilm production by C.albicans in a microtiter plate after $\mathrm{CV}$ staining.

\section{RESULTS}

CHRO Magar Candida accurately distinguished $>92 \%$ of our Candida strains. After identifying biochemically by API AUX Candida, a total of 300 isolates of C.albicans were used to test forin vitro antifungal susceptibility against planktonic C.albicans and then its biofilm production. Planktonic antifungal susceptibility was proceeded disk diffusion procedure which appeared that C.albicans was susceptible voriconazole in $240(80 \%)$ and fluconazole in $195(65 \%)$ isolates. C. albicans was found susceptible dose dependent (SDD) voriconazole in $21(7 \%)$ and fluconazole in $28(9 \%)$ isolates respectively. Seventy-seven (26\%) and 38 (13\%) isolates of C.albicans were resistant voriconazole and fluconazole, respectively (fig-3).

Sessile antifungal susceptibility of C.albicans isolates was performed through broth microdilution procedure, in which 161 (54\%) isolates had susceptible, $36(12 \%)$ were SDD and 148 (49\%) haveresistant to fluconazole, and $160(53 \%)$ isolates were susceptible, 42 $(14 \%)$ were SDD and 98 (33\%) were resistant to voriconazole (fig-4). Among 300 C.albicans, 285 (95\%) were biofilm producer and $15(5 \%)$ were not biofilm producer

Level of significance was applied between the two variables i.e. among fluconazole and voriconazole for plankton and sessile C.albicans as shown in table.

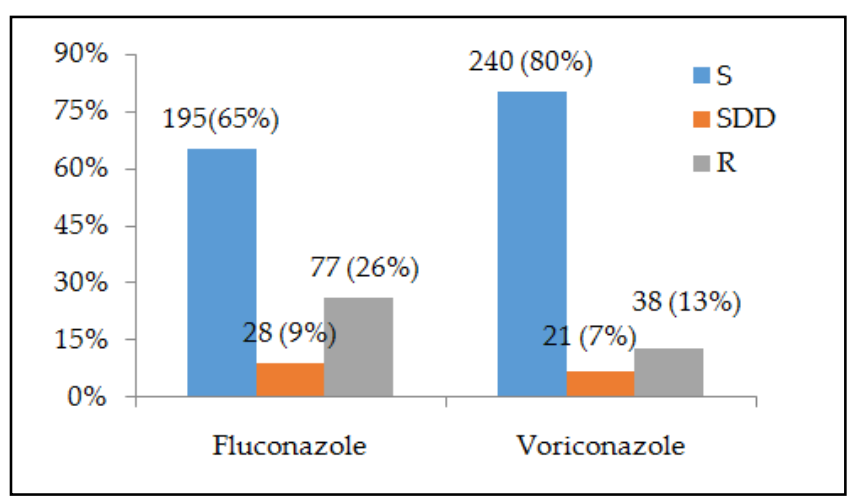

Figure-3: Planktonic antifungal susceptibility of fluconazole and voriconazole by disk diffusion procedure.

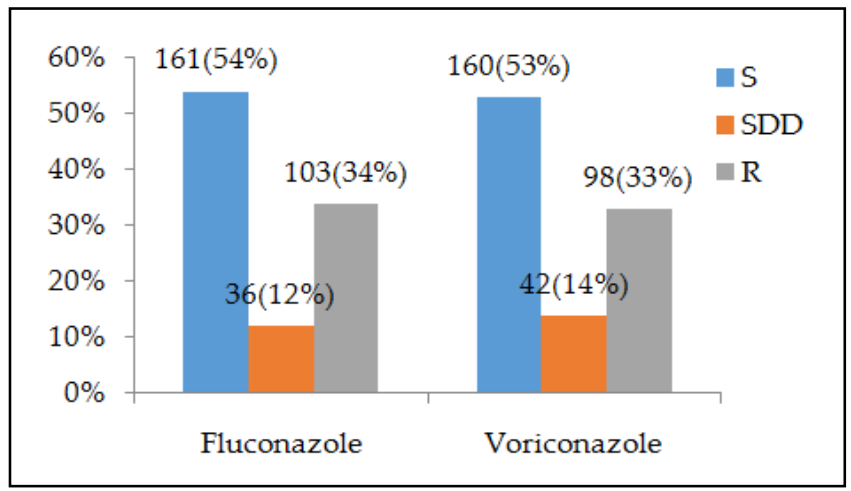

Figure-4: Sessile antifungal susceptibility against fluconazole and voriconazoleby microdilution method.

\section{DISCUSSION}

A proper and prompt diagnosis of C.albicans infection as a pathogenic yeast playsa major role for its definitive treatment. Germ tube test and Gram staining are still the easiest and reliable methods for the recognition of Candida spp. CHRO Magar Candida is used to be a rapidand reliable procedure for recognition of Candida species as compared to API $20 \mathrm{C} \mathrm{AUX}^{15}$. CHRO Magar Candida correctly identified $92 \%$ of our C.albicans strains which is in consistence with the result of astudy done by a researcher in $2012^{16}$.

Forthe treatment of superficial or systemic Candida infections, the important classes of antifungal drugs used are azoles, echinocandins and polyenes ${ }^{17}$. Azoles were used here because they are reliable and easilyaccessible antifungal agents. Fluconazole and

Table: Correlation between plankton and Sessile antifungal susceptibilityin clinical isolates.

\begin{tabular}{l|c|c|c|c|c|c|c}
\hline & \multicolumn{3}{|c|}{ Fluconazole } & \multicolumn{3}{c|}{ Voriconazole } & R-value \\
\cline { 2 - 8 } & Sensitive & SDD & Resistance & Sensitive & SDD & 22 & 38 \\
\hline $\begin{array}{l}\text { Plankton antifungal } \\
\text { susceptibility test }\end{array}$ & 195 & 28 & 77 & 240 & 42 & 90.01 \\
\hline $\begin{array}{l}\text { Sessile antifungal } \\
\text { susceptibility test }\end{array}$ & 161 & 36 & 103 & 160 & 42 & $<0.01$ \\
\hline
\end{tabular}


voriconazole are mostly used to treat candida or other fungal infections due to their good fungi static effect. Their action is by blocking the ergo sterol production and targeting the enzyme lanosterol 14-demethylase (related to the ERG11 gene), thereby, leads tocollection of intermediates of toxic sterol pathway ${ }^{18}$.

Our results of antifungal susceptibility appeared significantly lower susceptibility of azole antifungal agents againstboth planktonic and sessile C.albicans like other studies. Results of planktonic antifungal susceptibility appeared that fluconazole was susceptible against 195 (65\%) and that of voriconazole was against $241(80 \%)$ C.albicans isolates ${ }^{19}$. The susceptibility of voriconazole against C.albicans is higher in most of the studies as voriconazole is a novel azole with more strict binding to the sterol 14 a-demethylase, thereby more constructive inhibiting ergo sterol synthesis ${ }^{20}$. Twenty-eight (9\%) and 21 (7\%) isolates of C.albicans were showed that it were susceptible dose-dependent (SDD) to fluconazole and voriconazole, respectively. Seventy-seven (26\%) and $38(13 \%)$ isolates were found resistant to fluconazole and voriconazole respectively. One of the study was carried out in the United Kingdom, on different confines of Candida species, shows comparable figures of $206(76 \%)$ C.albicans isolates as susceptible, 25 as SDD and $39(14 \%)$ as resistant to fluconazole by using CLSI-validated methods. A similar study carried out in Singapore and China found that $28.1 \%$ of Candida confines were susceptible, $8.4 \%$ were SDD, while $63.6 \%$ confines were resistant to fluconazole.

Antifungal susceptibility testing of planktonic cells against fluconazole and voriconazole was performed by NCCLS M-27A broth microdilution by doing MICs in this study. In a similar study, $74 \%$ of C.albicans isolated from blood stream were resistant to fluconazole and $8.5 \%$ were resistant to voriconazole ${ }^{21}$.

Biofilms are more commonly formed adherent to solid surfaces can also be produced even in edges of liquid to air. Aquatic environments, biomaterials, artificial structures and mammalian tissuesand plant are the environments most commonly colonized by the biofilms. Almost all species of C.albicanspossess the capability of biofilm formation. Candida biofilm resistance phenomenon was for the first explained in 1995 for C.albicans by Hawser and Douglas (1995). Lower resistance to azole antifungals has been shown in planktonic conditions as compared to C.albicans biofilm experimentally, as shown in our study ${ }^{2}$. Biofilm formation with associated antifungal resistance is increasing by the yeardue to increase in the use of indwelling me- dical devices. Our data found that $95 \%$ of all the isolated Candida albicanswere biofilm producer, which was in conformance with a study in 2017 showing 90\% of all Candida albicans as biofilm producer. The slight increase in biofilm production in this study is attributable out being clinical specimens.

\section{RECOMMENDATIONS}

Candida Biofilm producing infections are an emerging problem. Using the variables for biofilm production, needed an intervention strategies can be implemented to lower biofilm-based Candidiasis, as there is a high antifungal resistance watched in biofilm-producing strains in this study. There is also a need to search for newerand effective antifungal agents with potent anti-biofilm abilities due to the increasing resistance to conventional antifungal drugs like azoles, and also due to their high probability of biofilm formation on implanted medical devices and tissues.

\section{CONCLUSION}

Planktonic C.albicans clinical confines were more susceptible to voriconazole than fluconazole and sessile isolates. Biofilm formation was very high among all the isolates of C.albicans.

\section{CONFLICT OF INTEREST}

This study has no conflict of interest to be declared by any author.

\section{REFERENCES}

1. Clancy CJ, Nguyen MH. Finding the "missing 50\%" of invasive candidiasis: how nonculture diagnostics will improve understanding of disease spectrum and transform patient care. Clin Infec Dis 2013; 56(9): 1284-92.

2. Leroy G, Lambiotte F, Thévenin D, Lemaire C, Parmentier E, Devos P, et al. Evaluation of" Candida score" in critically ill patients: a prospective, multicenter, observational, cohort study. Annals Inten Care 2011; 1(1): 50-55.

3. Moazeni M, Kelidari HR, Saeedi M, Morteza-Semnani K, Nabili M, Gohar AA, et al. Time to overcome fluconazole resistant Candida isolates: solid lipid nanoparticles as a novel antifungal drug delivery system. Colloids Surf. B, Biointerfaces 2016; 142(1): 400-407.

4. Spanu T, Posteraro B, Fiori B, D'Inzeo T, Campoli S, Ruggeri A, et al. Direct MALDI-TOF mass spectrometry assay of blood culture broths for rapid identification of Candida species causing bloodstream infections: an observational study in two large microbiology laboratories. J Clinical Microbiol 2012; 50(1): 176-79.

5. Hage CA, Carmona EM, Epelbaum O, Evans SE, Gabe LM, Haydour $Q$, et al. Microbiological laboratory testing in the diagnosis of fungal infections in pulmonary and critical care practice. An official American Thoracic Society clinical practice guideline. Am J Respirat Critical Care Med 2019; 200(5): 535-50.

6. Nguyen MH, Wissel MC, Shields RK, Salomoni MA, Hao B, Press EG, et al. Performance of Candida real-time polymerase chain reaction, $\beta$-D-glucan assay, and Blood cultures in the diagnosis of invasive candidiasis. Clin Infec Dis 2012; 54(9): 1240-48.

7. Souza ACR, Fuchs BB, Pinhati HM, Siqueira RA, Hagen F, Meis $\mathrm{JF}$, et al. Candida parapsilosis resistance to fluconazole: mole- 
cular mechanisms and in vivo impact in infected Galleria mellonella larvae. Antimicrob Agents chemother 2015; 59(10): 6581-87.

8. Kakakhel MA, Wu F, Gu J-D, Feng H, Shah K, Wang W. Controlling biodeterioration of cultural heritage objects with biocides: A review. Inter Biodeterioration \& Biodegradation 2019; 143(1): 104721-25.

9. Suh S-O, Zhou JJ. Methylotrophic yeasts near Ogataea (Hansenula) polymorpha: a proposal of Ogataea angusta comb. nov. and Candida parapolymorpha sp. nov. FEMS Yeast Res 2010; 10(5): 631-38.

10. Pappas PG, Kauffman CA, Andes DR, Clancy CJ, Marr KA, Ostrosky-Zeichner L, et al. Clinical practice guideline for the management of candidiasis: 2016 update by the Infectious Diseases Society of America. Clin Infec Dis 2016; 62(4): e1-e50.

11. Mc Carty TP, Pappas PG. Invasive candidiasis. Infec Dis Clin 2016; 30(1): 103-24.

12. Chen S, Tong Z, Lee O, Halliday C, Playford E, Widmer F, et al. Clinician response to Candida organisms in the urine of patients attending hospital. Europ J Clin Microbiol Infec Dis 2008; 27(3): 201-08.

13. Pappas PG, Lionakis MS, Arendrup MC, Ostrosky-Zeichner L. Invasive candidiasis. Nature Rev Dis Prim 2018; 4(1): 1-20.

14. https://www.calculator.net/sample-size-calculator.html? type $=1 \& \mathrm{cl}=99 \& \mathrm{ci}=1 \& \mathrm{pp}=50 \& \mathrm{ps}=194 \& \mathrm{x}=82 \& \mathrm{y}=15$.
15. Kullberg BJ, Arendrup MC. Invasive candidiasis. New Engl J Med 2015; 373(15): 1445-56.

16. Kollef M, Micek S, Hampton N, Doherty JA, Kumar A. Septic shock attributed to Candida infection: importance of empiric therapy and source control. Clin Infec Dis 2012; 54(12): 1739-46.

17. Andes DR, Safdar N, Baddley JW, Playford G, Reboli AC, Rex $\mathrm{JH}$, et al. Impact of treatment strategy on outcomes in patients with candidemia and other forms of invasive candidiasis: a patient-level quantitative review of randomized trials. Clin Infec Dis 2012; 54(8): 1110-22.

18. Kullberg B, Verweij P, Akova M, Arendrup M, Bille J, Calandra $\mathrm{T}$, et al. European expert opinion on the management of invasive candidiasis in adults. Clinical Microbiol Infec 2011; 17(1): 1-12.

19. Reboli AC, Rotstein C, Pappas PG, Chapman SW, Kett DH, Kumar D, et al. Anidulafungin versus fluconazole for invasive candidiasis. New Engl J Med 2007; 356(24): 2472-82.

20. Charles PE, Castro C, Ruiz-Santana S, León C, Saavedra P, Martín E. Serum procalcitonin levels in critically ill patients colonized with Candida spp: new clues for the early recognition of invasive candidiasis. Inten Care Med 2009; 35(12): 2146-50.

21. Pfaller M, Boyken L, Hollis R, Messer S, Tendolkar S, Diekema D. Global surveillance of in vitro activity of micafungin against Candida: a comparison with caspofungin by CLSI-recommended methods. J Clin Microbiol 2006; 44(10): 3533-38. 